\title{
Bridge to Transplantation: Current Outcomes
}

\author{
Sanjeev Aggarwal, M.D. and Francis D. Pagani, M.D., PhD. \\ Division of Thoracic and Cardiovascular Surgery, University of Louisville, Louisville, Kentucky \\ and Section of Cardiac Surgery, University of Michigan Health System, Ann Arbor, Michigan
}

\begin{abstract}
AвStract The use of implantable ventricular assist devices for the treatment of advanced, decompensated heart failure as a bridge to heart transplantation has experienced considerable evolution over the past decade. Improvements in the management and prevention of serious adverse events, and introduction of new continuous-flow rotary pumps that are smaller, more durable, and associated with fewer serious adverse advents has expanded the use of this therapy to a larger population of patients for bridge to transplant indication. doi: 10.1111/j.1540-8191.2010.00996.x (J Card Surg 2010;25:455-461)
\end{abstract}

\section{BACKGROUND}

Heart transplantation remains the most successful treatment option for patients with advanced heart failure. ${ }^{1}$ As a consequence of limited donor availability, mechanical circulatory support (MCS) in the form of implantable ventricular assist device (VAD) therapy has become an established treatment for patients with advanced heart failure as a bridge to transplantation (BTT). ${ }^{2-4}$ The successful clinical use of a ventricular assist device (VAD) as BTT therapy began in the early 1980s. ${ }^{5}$ Donald Hill first reported the successful utilization of the pneumatically actuated PierceDonachy paracorporeal device as a BTT. ${ }^{5}$ In that same year, Philip Oyer of Stanford University successfully implanted the Novacor left ventricular assist device (LVAD) as a BTT. ${ }^{6}$ The Novacor device, developed by Peer Portner, was the first utilization of an electrically powered implantable LVAD. Victor Porier and colleagues at Thermo Cardiosystems, Inc. developed the HeartMate IP1000, an implantable pneumatic, pulsatile device that utilized a textured-blood contacting surface that promoted cellular in-growth on the surface and eliminated the need for anticoagulation therapy. ${ }^{2,5}$ O.H. Frazier of the Texas Heart Institute implanted this device successfully as a BTT in $1988 . .^{5}$ The advantages of an implantable VAD versus medical therapy as a BTT were initially demonstrated in a multicenter clinical trial of the HeartMate IP 1000 in 1992. ${ }^{2}$ In 1994, the Food and Drug Administration (FDA) approved the use of the pneumatically driven HeartMate IP 1000 implantable

Address for correspondence: Francis D. Pagani, M.D., PhD., Otto Gago, M.D., Professor of Surgery, Section of Cardiac Surgery, University of Michigan Health System, Cardiovascular Center-Room 5161, 1500 East Medical Center Drive, SPC 5864, Ann Arbor, MI 481095864. Tel: 734-647-2894; e-mail: fpagani@umich.edu

Disclosures: Dr. Pagani is a principal site investigator for clinical trials evaluating the HeartMate II (Thoratec Corporation), DuraHeart (Terumo Cardiovascular Corporation), and HVAD (HeartWare Corporation) devices.
VAD as a BTT. Further clinical evaluation of the Novacor LVAD and HeartMate VE (electrical-powered version of the HeartMate IP 1000) LVAD led to approval of these systems for BTT therapy by the FDA in 1998. These systems represented the first wearable left ventricular assist systems for BTT therapy that permitted hospital discharge and outpatient management.

Direct randomized comparisons of VAD therapy to medical therapy for BTT have never been performed because of the ethical concerns for withholding potentially life-saving therapy. However, VAD therapy provides significant survival benefit and functional improvement compared to historical experiences or to concurrent nonrandomized control patients treated with medical therapy. ${ }^{2,3}$ Frazier et al. reported on 280 patients implanted with the HeartMate VE LVAD for BTT therapy. ${ }^{3}$ Of the 280 patients, $71 \%$ survived long term with $67 \%$ surviving to heart transplantation and $4 \%$ surviving to device explantation for myocardial recovery. However, adverse events associated with LVAD therapy utilizing the first generation of large pulsatile devices were significant, and included bleeding (31\% of patients), infection (40\% of patients), neurologic dysfunction ( $5 \%$ of patients), thromboembolic events ( $6 \%$ of patients), and major failure of the device (1\% of patients). Survival on device support was approximately $50 \%$ at one year, and posttransplant survival was $84 \%$ at one year. Similar results were also reported for the Novacor LVAD by Dagenais et al. ${ }^{7}$ and Deng et al. ${ }^{8}$ although this device demonstrated significantly enhanced long-term durability and reliability over the HeartMate VE LVAD. In an analysis of outcomes from patients implanted with the Novacor LVAD in the Novacor European Registry, survival was approximately $60 \%$ at one year for a cohort of 276 patients with lower-risk characteristics at implant, and approximately $20 \%$ at one year for 90 patients with high-risk characteristics at implant that included respiratory failure with septicemia, preexisting right heart failure, older age (>65 years), acute postcardiotomy 
etiology, and presentation with acute myocardial infarction. ${ }^{8}$ Major adverse events included bleeding, right heart failure, respiratory infection, renal failure, stroke, device pocket infection, and bacteremia. Sepsis was the leading cause of death followed by organ failure, bleeding, stroke, and other causes.

In addition to survival benefit, early studies of VAD therapy have demonstrated improved recovery of organ dysfunction, reversal of neurohormonal activation, and improved exercise performance and rehabilitation as compared to medical therapy. ${ }^{3,9-12}$ Improvements in the cellular biology of the myocardium and overall improvement in left ventricular function also have been reported following VAD therapy leading to the potential use of VAD therapy as a bridge to recovery and obviating the need for heart transplantation. ${ }^{13,14}$ These combined experiences and observations have led to a significant increase in the number of patients BTT over the past decade.

\section{CURRENT OUTCOMES WITH VAD THERAPY FOR BTT INDICATION}

First-generation pulsatile, volume displacement devices, until recently, were the mainstay of VAD therapy for BTT with over 4000 devices implanted worldwide. However, these devices have manifest significant limitations in their design that preclude their practical use for extended periods of MCS as a BTT or as a permanent replacement for destination therapy (DT). As waiting times for heart transplantation have increased, extended periods of MCS highlighted limitations in the design reliability and durability of pulsatile pumps. ${ }^{15}$ These limitations included a large pump size restricting mobility, requirement for extensive surgical dissection for implant leading to bleeding and infection complications, a large body habitus of the recipient limiting options for device therapy for women, the presence of a large-diameter percutaneous lead for venting air, and audible pump operation. 3,7 A critical limitation of the these devices, particularly the HeartMate VE device and the subsequent later model, the HeartMate XVE, was the high incidence of reoperation for device exchange for device malfunction. ${ }^{15,16}$ Reports on the HeartMate VE and XVE have demonstrated a greater than $50 \%$ rate of device failure at two years. ${ }^{15,16}$ The development of continuous-flow (CF) rotary pump technology represents an innovative design for VAD therapy and has now largely replaced the use of large pulsatile volume replacement pumps such as the HeartMate XVE and Novacor devices. ${ }^{17,18}$ The CF rotary devices have the advantage of a smaller pump size and potential for greater mechanical reliability by simplification of the pumping mechanism. Reports from clinical trials of these newer pump designs with axial blood flow have demonstrated efficacy in providing hemodynamic support and improvements in functional capacity and quality of life. ${ }^{17,18}$

The HeartMate II LVAD is a CF rotary pump with axial design that is representative of the second generation of LVAD technology in clinical use in the United States. ${ }^{17,18}$ Other second generation rotary

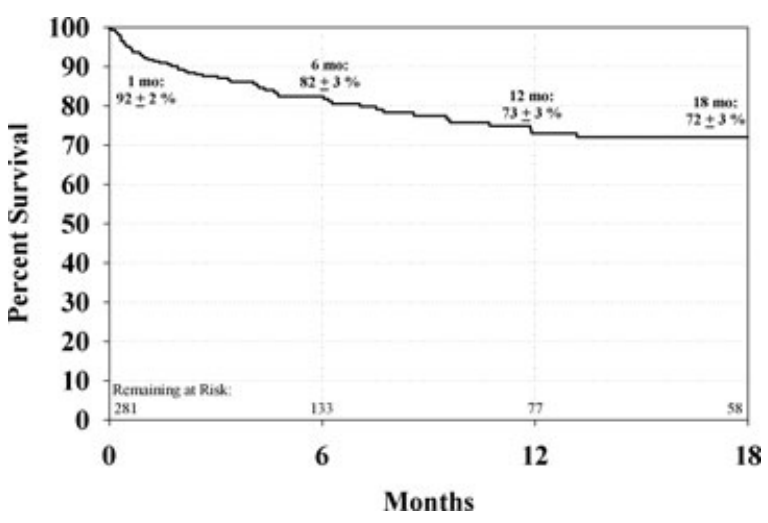

Figure 1. Survival analysis for patients receiving mechanical circulatory support with a continuous-flow left ventricular assist device (axial flow design) censored at the time of heart transplantation and device explantation for cardiac recovery. ${ }^{18}$

pump technology with axial blood flow design include the DeBakey LVAD ${ }^{19}$ and Jarvik LVAD. 20,21 The HeartMate II LVAD recently completed an FDA evaluation for BTT therapy in the United States in a cohort of 133 patients. ${ }^{17}$ Since this report, 336 additional patients have undergone implantation of the HeartMate II LVAD as of April 2008 through a continued-access protocol approved by the FDA. To date, over 400 patients have been evaluated with the HeartMate II LVAD for BTT therapy and the device has received approval by the FDA for BTT indication in May of 2008. In an extended follow-up report of 281 patients evaluated for BTT therapy with the HeartMate II device, 222 (79\%) either received a transplant, recovered cardiac function and underwent device explantation, or remained alive with ongoing LVAD support at 18-month follow-up. ${ }^{18}$ At 18 months, 157 (55.8\%) patients had received a heart transplant, 58 (20.6\%) remained alive with ongoing LVAD support, 56 (19.9\%) patients died, seven $(2.5 \%)$ patients recovered cardiac function and underwent device explantation, and three (1\%) patients were withdrawn from the study after device explantation and exchange for another type of LVAD. Overall survival for the patients who continued on LVAD support was $82 \%$ (95\% confidence interval [Cl]: $77 \%$ to $87 \%$ ) at six months, $73 \%$ (95\% Cl: $66 \%$ to $80 \%)$ at one year, and $72 \%(95 \% \mathrm{Cl}: 65 \%$ to $79 \%)$ at 18 months (Fig. 1). Of the 157 patients that received a transplant, posttransplant survival was $96 \%$ at 30 days and $86 \%$ at one year. The primary causes of death were sepsis in 11 patients (4\%), stroke in 10 (4\%; ischemic: $n=5,2 \%$; hemorrhagic: $n=5,2 \%$ ), and right heart failure in seven (3\%). Other causes included device-related deaths in seven patients $(3 \%)$, multiorgan failure in five $(2 \%)$, anoxic brain injury in three $(1 \%)$, bleeding in three $(1 \%)$ and other causes in ten (4\%; cancer, respiratory failure, hyperthermia, air embolism, and unknown). There were a total of seven device-related deaths, four that were attributed to malfunction of implanted components (pump thrombosis in two, inflow graft that was twisted during implantation in one, and outflow elbow 
disconnect in one), and three that were attributed to external components (severed percutaneous lead in one, power loss in two). Only 13 of the deaths in the 281 (4.6\%) patients (or $23 \%$ of the 56 deaths) occurred after six months of device. Bleeding requiring transfusion and surgery were the most common adverse events in the study and were primarily observed within the first 30 days of device implantation. Stroke was observed in 25 (8.9\%) patients, with the event rate greatest in the first 30 days. Ischemic strokes were more common than hemorrhagic strokes. After the first 30 days, the event rates for ischemic and hemorrhagic stroke were 0.05 and 0.03 events per patientyear, respectively. Six (2\%) additional patients had transient ischemic attacks that completely reversed. Localized infection not related to the device occurred in 84 (30\%) patients. Infection associated with the percutaneous lead was observed in 41 (14\%) patients, and there were five ( $2 \%$ ) preperitoneal pump pocket infections. Respiratory and renal failure occurred in 72 $(26 \%)$ and $30(11 \%)$ patients, respectively. Thirty-six $(13 \%)$ patients had right heart failure requiring inotrope support for more than 14 days. Of the 53 (19\%) patients that developed postoperative right heart failure, survival with continued LVAD support at 18 months was $62 \pm 8 \%$. Twenty-six of these 53 (49\%) patients with right heart failure received a transplant. Seventeen $(6 \%)$ patients received temporary support with right $\mathrm{VADs}$ (median time of support 11.5 days; range 0 to 148 days). The median duration of postoperative inotrope support was nine days. A total of 11 (4\%) patients underwent 12 HeartMate II LVAD replacements for either device thrombosis ( $n=4 ; 1.4 \%$; two of whom subsequently died), surgical complications at the time of LVAD implantation ( $n=3 ; 1.1 \%$ ), percutaneous lead wire damage ( $n=4 ; 1.4 \%$ ), or for development of device infection ( $n=1 ; 0.3 \%$ ). One patient had two of the pump replacements (thrombosis and infection). Of the 12 pump replacements, nine (75\%) were replaced with another HeartMate II LVAD, and the patients continued in the study, and three $(25 \%)$ were replaced with another type of LVAD device, and the patients were withdrawn from the study. There were no failures of the mechanical pumping mechanism. The median time to pump replacement was 106 days (range 0 to 672 days). The freedom from major device malfunction resulting in death $(n=4)$ or device replacement for all causes (malfunction, thrombosis, or infection; $n=9$ without deaths) was $96 \%(95 \% \mathrm{Cl}: 95 \%$ to 99\%) at six months, $93 \%(95 \% \mathrm{Cl}: 90 \%$ to $98 \%)$ at one year, and $92 \%(95 \% \mathrm{Cl}: 88 \%$ to $97 \%)$ at 18 months (Fig. 2). Functional assessment with six-minute walk and NYHA functional classification were performed for patients remaining on device support up to six months. Of 109 patients with paired values at baseline and at six months, only 14 of 109 (13\%) were able to perform a six-minute walk test at baseline, compared with 97 (89\%) patients after six months of support, and there was a significant improvement in distance walked between baseline and six months. Additionally, NYHA functional classification improved from $3.9 \pm 0.3$ at baseline (with $0 \%$ of patients in NYHA functional class

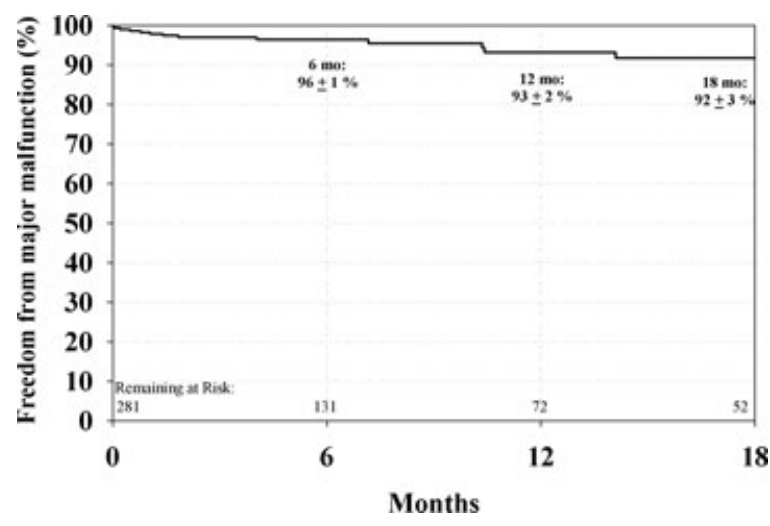

Figure 2. Analysis of freedom from major device malfunction as defined as the freedom from death as the result of major device malfunction or need for device replacement for all causes including device malfunction, thrombosis, or infection. $^{18}$

I or II) to $1.8 \pm 0.7$ at six months (with $83 \%$ in functional class I or II). Quality of life assessed by both the Minnesota Living With Heart Failure and the Kansas City Cardiomyopathy Questionnaires was significantly improved at six months compared with baseline, with mean scores improving over $25 \mathrm{U}$, or $41 \%$ and $75 \%$, respectively. Hepatic (total bilirubin, serum aspartate aminotransaminase, and serum alanine aminotransaminase) and renal (blood urea nitrogen) function significantly improved from baseline to six months, but changes in serum creatinine were not statistically significant.

\section{INFLUENCE OF VAD THERAPY ON POSTTRANSPLANT OUTCOMES}

Although VAD therapy significantly improves survival to transplant for patients with advanced decompensated heart failure, it has not been clear whether patients undergoing transplant with prior VAD support have improved or decreased survival compared to patients undergoing transplant without prior VAD support. Studies to date have described an adverse, neutral, or beneficial effect of implantable VAD therapy on posttransplant survival. ${ }^{22-26}$ The differences in findings of these studies are influenced by inclusion of patients supported by short-term extracorporeal MCS devices not intended for BTT indication, single center versus multicenter reports, and whether posttransplant survival following support VAD support was compared to all transplant patients (UNOS status 1 and 2) or UNOS status $1 \mathrm{~A}$ or $1 \mathrm{~B}$ patients with inotrope therapy alone. The use of short-term extracorporeal VADs for MCS as a BTT adversely influences early posttransplant survival compared to patients supported with implantable VADs at the time of transplantation. ${ }^{24,26}$ This observation is likely attributed to the improved functional status and improved status of organ function for patients on implantable VAD support at the time of transplant compared to patients supported with shortterm extracorporeal VADs. In a recent analysis of the United Network for Organ Sharing Thoracic Registry, 
Patlolla et al. ${ }^{26}$ reported that the use of intracorporeal VADs was associated with a small increase in mortality in the first six months and a clinically significant but small $(2 \%)$ increase in mortality beyond five years. However, the reasoning for a late adverse effect on posttransplant survival from the pretransplant use of VAD therapy is not well understood nor validated from other studies of long-term transplant outcomes.

\section{INTERAGENCY REGISTRY FOR MECHANICALLY ASSISTED CIRCULATORY SUPPORT}

The Interagency Registry for Mechanically Assisted Circulatory Support (INTERMACS) database, funded by the United States National Heart, Lung and Blood Institute (NHLBI), is a registry for patients who receive implantable and durable VAD devices that are approved by the FDA. ${ }^{27,28}$ The INTERMACS registry represents one of the largest available data repositories for the study of VAD outcomes intended for BTT or DT. The most recent published data analysis of MCS outcomes for VAD therapy reviews the first 18 months of data entry into the registry and almost exclusively represent outcomes from patients undergoing implantation with the first generation of pulsatile, volume displacement technology such as the HeartMate XVE, Thoratec post-VAD (pVAD), Thoratec IVAD, and Novacor LVAD. ${ }^{28}$ Survival with VAD support at one year was approximately $60 \%$ for all patients and significantly worse for those patients requiring biventricular assist device (BiVAD) support or support with a total artificial heart compared to patients requiring LVAD support alone. ${ }^{27,28}$ (Figs. 3 and 4) Patients undergoing implantation of a device in the presence of critical cardiogenic shock (i.e., INTERMACS level 1) had worse outcomes compared to device implantation in patients with more stable forms of advanced heart failure (INTERMACS levels 2 through 7$)^{28}$ (Fig. 5). These observations highlight the importance of proper patient selection and

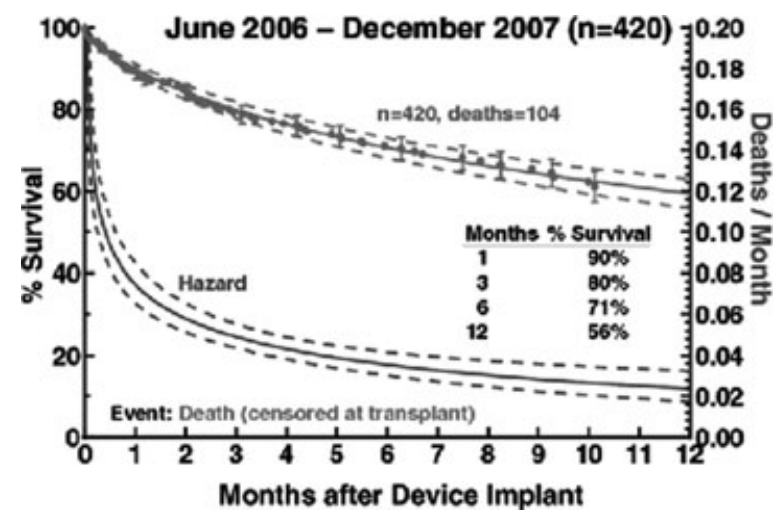

Figure 3. Survival analysis for patients on mechanical circulatory support and entered into the INTERMACS Registry from June 2006 through December 2007. (Figure obtained from the INTERMACS Registry website: http://WwW. uab.edu/ctsresearch/intermacs/presentations.htm and previously presented at the International Society of Heart and Lung Transplantation, 28th Meeting and Scientific Sessions, April 2008. $)^{28}$

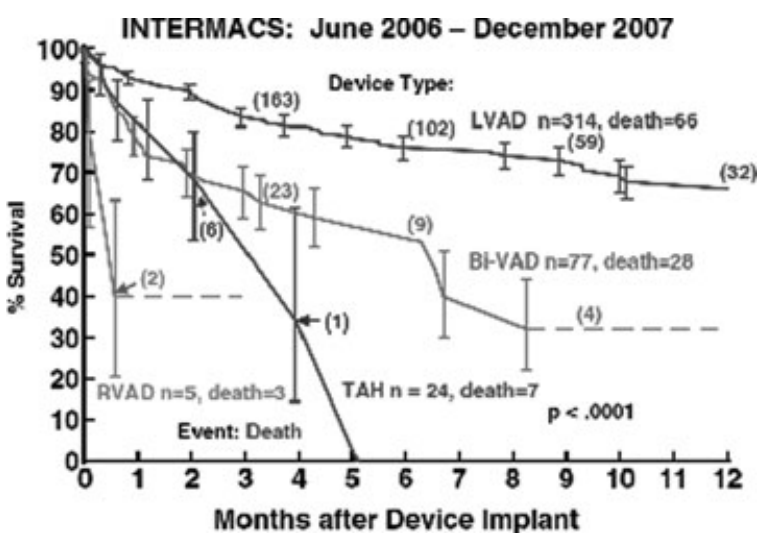

Figure 4. Survival analysis of patients on mechanical circulatory support and entered into the INTERMACS Registry from June 2006 through December of 2007 and stratified by device location. ( $L V A D=$ left ventricular assist device; $R V A D=$ right ventricular assist device; BiVAD = biventricular assist device; TAH = total artificial heart). (Figure obtained from the INTERMACS Registry website: http://www. uab.edu/ctsresearch/intermacs/presentations. htm and previously presented at the International Society of Heart and Lung Transplantation, 28th Meeting and Scientific Sessions, April 2008. $)^{28}$

timing of initiation of VAD therapy on pVAD outcomes. Patients with significant organ dysfunction at the time of LVAD implant, accompanied by a greater degree of hemodynamic compromise, have a significantly higher risk of requiring BiVAD support, higher risk of major adverse events, and significantly higher risk of death during VAD support. ${ }^{2-4}$ Thus, hemodynamic stability provided with inotrope support, INTERMACS level 3, appears to be a reasonable threshold and indication for LVAD therapy. This clinical threshold appears to maximize post-LVAD survival. Implantation of a device for less advanced stages of heart failure, INTERMACS

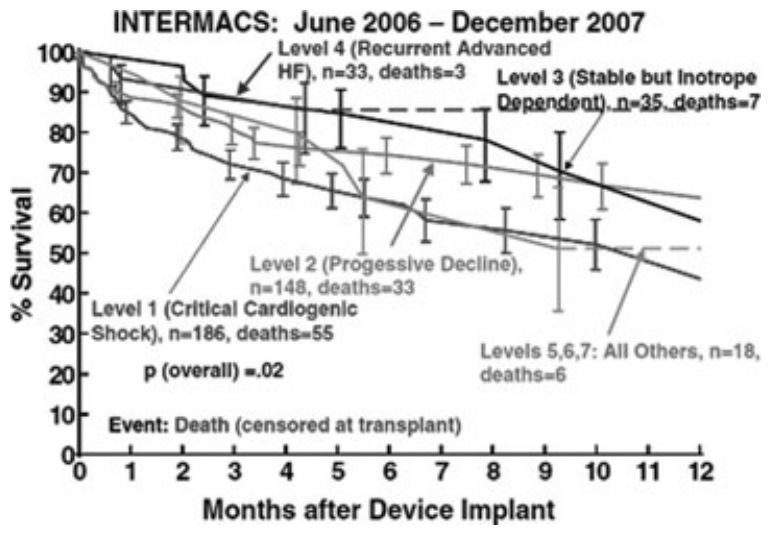

Figure 5. Survival analysis of patients on mechanical circulatory support and entered into the INTERMACS registry from June 2006 through December 2007 stratified by acuity of illness assessed by the INTERMACS patient profile. (Figure obtained from the INTERMACS Registry website: http://www. uab.edu/ctsresearch/intermacs/presentations.htm and previously presented at the International Society of Heart and Lung Transplantation, 28th Meeting and Scientific Sessions, April 2008. $)^{28}$ 
levels 4 through 7, does not appear to offer survival advantage over implantation of a device at INTERMACS level 3. Prospective, randomized studies will likely be required to determine the benefit of LVAD therapy at less advanced stages of heart failure.

Since May of 2008, following FDA approval of HeartMate II LVAD, an increasing number of patients entered into the INTERMACS registry have received VAD support with the new generation of CF rotary technology. Subsequent reports from the INTERMACS registry will highlight outcomes from new CF rotary pump technology and offer unique insight with comparison to older technology for BTT indication.

\section{NEWER DEVICE DESIGNS FOR BTT THERAPY}

Although significant improvements in pump design have occurred with the second generation of CF rotary pumps (axial design), there remain a number of potential concerns with this technology. The presence of contact bearings to suspend the rotor represents a potential point of frictional wear resulting in device failure and subsequent need for device exchange. The second-generation pump technology still demonstrates the potential for thrombus formation on the device rotor and bearing interface due to the presence of stasis and incomplete bearing wash. ${ }^{17,29}$ However, the concern for development of thrombus on the rotor and bearing contact points has varied significantly with different second-generation rotary pumps. ${ }^{29}$ The presence of stators to suspend and redirect blood flow also represents an obstruction within the blood flow path. Thrombosis from blood stasis can be caused by blood flow disturbances and recirculating zones associated with the supports required by contact bearing design. Clinical studies have documented the problem with device thrombus requiring device exchange or treatment with thrombolytic therapy, and have also shown a reduced but persistent risk of stroke. ${ }^{17,29} \mathrm{~A}$ significant proportion of strokes reported during clinical trials with second-generation devices have occurred early following surgery and were associated with the implant procedure, likely representing air or particulate emboli possibly from sources other than the device. The proportion of strokes that are attributable to transient thrombus formation within the pump or thrombus formation within the heart is unknown. In addition, this technology still requires long-term antithrombotic therapy and subsequently, hemorrhagic complications are observed with this therapy. The designation of the new third generation of rotary pumps has generally been used to categorize CF rotary devices with an impeller or rotor suspended in the blood flow path using a noncontact-bearing design. ${ }^{30}$ In the majority of circumstances this design utilizes a "centrifugal" blood flow path and incorporates either magnetic and/ or hydrodynamic levitation of the internal impeller. Currently, CF pumps with centrifugal and noncontact-bearing design in clinical trial evaluation in the United States include the DuraHeart LVAD (Terumo Heart Corporation, Ann Arbor, MI, USA) and HVAD (HeartWare Corporation, Miami, FL, USA). ${ }^{31-33}$
The DuraHeart LVAD is a CF rotary pump with centrifugal and noncontact-bearing design. ${ }^{32}$ The device has a displacement volume of $180 \mathrm{~cm}^{3}$ and a weight of $540 \mathrm{~g}$. Its external dimensions are $72 \mathrm{~mm}$ in width and $45 \mathrm{~mm}$ in height. The pumping unit consists of an upper housing with the levitation system, impeller, and bottom housing containing the external-drive motor. The device is designed with active magnetic levitation of the impeller along with hydrodynamic bearings to support impeller levitation in case of failure of the magnetic levitation system. The impeller is rotated through magnetic coupling between permanent magnets embedded on the motor side of the impeller and an external-drive motor that utilizes a bearing design. Three electromagnets and three position sensors are mounted in the upper housing. Tilting and axial displacements of the impeller are monitored and controlled using a three-degrees-of-freedom control. The ferromagnetic ring on the opposite side of the impeller is levitated by the electromagnet, and position sensors control the impeller so that it is always positioned at the center of the blood chamber. Radial impeller movement is passively suspended with a bias flux through electromagnetic rotor and drive magnet rotor.

Clinical evaluation of the DuraHeart device has recently concluded in Europe, and clinical evaluation of the device in the United States began in July of 2008. A preliminary report of the European experience was recently presented at the International Society of Heart and Lung Transplantation in April of 2008. ${ }^{32}$ Thirty-five patients with advanced heart failure (NYHA class IV, 14 ischemic, five females) who were eligible for heart transplantation underwent implantation of the DuraHeart device from January of 2004 through September of 2007. Median age of the patients was 56 (range 29 to 73) years with a median body surface area of 1.9 (1.4 to 2.4) $\mathrm{m}^{2}$. The average duration of device support was $330 \pm 220$ (17 to 808) days with a cumulative duration of 21 years. Fourteen patients (40\%) underwent heart transplantation at $194 \pm 146$ days. Nineteen patients (54\%) were supported for at least six months and seven (20\%) patients were supported for greater than one year. Fourteen patients (40\%) remain alive with ongoing device support (330 \pm 292 days). Kaplan-Meier survival at two years was $78 \%$. There were seven deaths (median time to deaths: 29 days). Six early deaths occurred for the initial 11 patients and four were associated with excessive anticoagulation/antiplatelet therapy that resulted in fatal intracerebral hemorrhage or subdural hematoma. After implementing less intensive anticoagulation and antiplatelet therapy comparable to mechanical heart valves, there was no ischemic or hemorrhagic stroke for the last 24 patients. Stroke-free survival for the last 24 patients was $94 \%$ at two years. Twenty-six patients $186 \%$ of one-month survivors) were discharged home, and the readmission rate was 1.5/pt-year. There was no pump mechanical failures, pump thrombosis, or hemolysis throughout the support duration.

The HVAD is a small CF rotary pump with centrifugal and noncontact-bearing design. ${ }^{33}$ The unique feature of the HVAD is its small design size. It has a 
displacement volume of $45 \mathrm{cc}$, and weighs $145 \mathrm{~g}$ with a flow capacity of up to $10 \mathrm{~L} / \mathrm{min}$. The HVAD impeller is suspended in place by combination of passive magnetic and hydrodynamic bearing systems to avoid mechanical contact and wear. The design integrates two motor stators for single-motor fault protection to increase reliability. The impeller suspension system uses a passive magnetic bearing for radial stiffness. Axial magnetic preload and hydrodynamic bearings on top of each impeller blade provide axial constraint.

The HVAD has undergone clinical evaluation in Europe and Australia and clinical evaluation in the United States began in May of 2008. In a multiinstitutional trial in Europe and Australia, 20 patients underwent implantation of the HVAD from March of 2006 through September of 2007. ${ }^{34}$ Mean age of the patients was $46 \pm 12$ (range 28 to 68 years) years. Median cardiopulmonary bypass time to implant the device was $67 \mathrm{~min}$ (range 21 to $140 \mathrm{~min}$ ). Mean duration of HVAD device support was $167 \pm 143$ days (range 13 to 425 days). Range of blood flow provided by the pump was 4.0 to $6.5 \mathrm{~L} / \mathrm{min}$. Three patients were successfully transplanted after 426, 349, and 157 days, respectively. One patient was weaned from pump support on postoperative day 266, two patients died on device (postoperative days 13 and 203), and 14 patients remain alive with ongoing device support. Actuarial survival at one year was $80 \%$.

\section{CONCLUSIONS}

Implantable VAD therapy is an established and viable treatment option for patients with advanced heart failure awaiting heart transplantation. Over the past decade, there has been a transition from pulsatile pumps to CF rotary pumps with axial and, more recently, centrifugal blood flow design. This transition to new technology has been associated with improvements in pump design contributing to a significant reduction in the occurrence of serious adverse events, particularly pump malfunction, device-related infection, and right heart failure. Bleeding, stroke, and thromboembolic events remain an important concern with VAD therapy. An improved understanding of the biology of the blood-device interface is necessary to understand optimal management of anticoagulation and device design to prevent these adverse events.

The majority of patients undergoing LVAD therapy as a BTT currently receive device intervention at significant stages of advanced, progressing or decompensated heart failure or cardiogenic shock (INTERMACS levels 1 and 2). This is obviously unavoidable for those patients presenting with acute hemodynamic change. However, current data suggest that intervention at less advanced stages, INTERMACS level 3 (stable on inotrope therapy), maximizes postimplant survival. Application of new device technology along with improved timing of intervention for those patients experiencing progressive decline will likely increase the overall effectiveness of LVAD therapy as a BTT.

\section{REFERENCES}

1. Mulligan MS, Shearon TH, Weill D, et al: Heart and lung transplantation in the United States, 1997-2006. Am J Transplant 2008;8:977-987.

2. Frazier O, Rose E, Macmanus $\mathrm{Q}$, et al: Multicenter clinical evaluation of the Heart Mate 1000 IP left ventricular assist device. Ann Thorac Surg 1992;53:1080-1090.

3. Frazier $\mathrm{OH}$, Rose $\mathrm{EA}, \mathrm{Oz} \mathrm{MC}$, et al: Multicenter clinical evaluation of the HeartMate vented electric left ventricular assist system in patients awaiting heart transplantation. J Thorac Cardiovasc Surg 2001;122:11861195.

4. Slaughter MS, Tsui SS, El-Banayosy A, et al: Results of a multicenter clinical trial with the thoratec implantable ventricular assist device. J Thorac Cardiovasc Surg 2007;133:1573-1580.

5. Joyce LD, Noon GP, Joyce DL, et al: Mechanical circulatory support-a historical review. ASAIO J 2004;50:x-xii.

6. Portner PM, Oyer PE, McGregor CGA, et al: First human use of an electrically-powered implantable ventricular assist system. Artif Organs 1985;9:36. [Abstract]

7. Dagenais F, Portner PM, Robbins RC, Oyer PE: The Novacor left ventricular assist system: Clinical experience from the Novacor registry. J Card Surg 2001;16:267-271.

8. Deng MC, Loebe M, El-Banayosy A, et al: Mechanical circulatory support for advanced heart failure: Effect of patient selection on outcome. Circulation 2001;103:231237.

9. Baughman KL, Jarcho JA: Bridge to life-cardiac mechanical support. N Engl J Med 2007;357:846-849.

10. Jaski BE, Lingle RJ, Kim J, et al: Comparison of functional capacity in patients with end-stage heart failure following implantation of a left ventricular assist device versus heart transplantation: results of the experience with left ventricular assist device with exercise trial. J Heart Lung Transplant 1999;18:1031-1040.

11. de Jonge N, Kirkels $H$, Lahpor JR, et al: Exercise performance in patients with end-stage heart failure after implantation of a left ventricular assist device and after heart transplantation: an outlook for permanent assisting? J Am Coll Cardiol 2001;37:1794-1799.

12. Haft J, Armstrong W, Dyke DB, et al: Hemodynamic and exercise performance with pulsatile and continuous flow left ventricular assist devices. Circulation 2007;116(Suppl I): $18-115$

13. Young JB: Healing the heart with ventricular assist device therapy: Mechanisms of cardiac recovery. Ann Thorac Surg 2001;71:S210-S219.

14. Burkhoff D, Klotz S, Mancini D: LVAD-induced reverse remodeling: Basic and clinical implications for myocardial recovery. J Card Failure 2006;12:227-239.

15. Dembitsky WP, Tector AJ, Park S, et al: Left ventricular assist device performance with long term circulatory support: Lessons from the REMATCH trial. Ann Thorac Surg 2004;78:2123-2130.

16. Pagani FD, Long JW, Dembitsky WP, et al: Improved mechanical reliability of the HeartMate XVE left ventricular assist system. Ann Thorac Surg 2006;82:1413-1419.

17. Miller LW, Pagani FD, Russell SD, et al: Use of a continuous-flow device in patients awaiting heart transplantation. N Engl J Med 2007;357:885-896.

18. Pagani FD, Miller LW, Russell SD, et al: Extended mechanical circulatory support with a continuous-flow left ventricular assist device. J Am Coll Cardiol 2009;54:312321

19. Goldstein DJ, Zucker M, Arroyo L, et al: Safety and feasibility trial of the MicroMed DeBakey ventricular assist 
device as a bridge to transplantation. J Am Coll Cardiol 2005;45:962-963.

20. Frazier $\mathrm{OH}$, Myers TJ, Jarvik RK, et al: Research and development of an implantable, axial-flow left ventricular assist device: the Jarvik 2000 heart. Ann Thorac Surg 2001;71(Suppl 1):S125-S132.

21. Siegenthaler MP, Frazier $\mathrm{OH}$, Beyersdorf $F$, et al: Mechanical reliability of the Jarvik 2000 Heart. Ann Thorac Surg 2006;81:1752-1758.

22. Aaronson KD, Eppinger M, Dyke BD, et al: LVAD therapy improves utilization of donor hearts. J Am Coll Cardiol 2002;39:1247-1254.

23. Massad MG, McCarthy PM, Smedira NG, et al: Does successful bridging with the implantable left ventricular assist device affect cardiac transplantation outcome? J Thorac Cardiovasc Surg 1996;112:1275-1283.

24. Taylor DO, Edwards LB, Boucek MM, et al: Registry of the International Society for Heart and Lung Transplantation: Twenty-third Official Adult Heart Transplantation Report-2006. J Heart Lung Transplant 2006;25:869-879.

25. Cleveland JC, Grover FL, Fullerton DA, et al: Left ventricular assist device as bridge to transplantation does not adversely affect one-year heart transplantation survival. J Thorac Cardiovasc Surg 2008;136:774-777.

26. Patlolla V, Patten RD, DeNofrio D, et al: The effect of ventricular assist devices on post-transplant mortality: An analysis of the United Network for Organ Sharing Thoracic Registry. J Am Coll Cardiol 2009;53:264-271.

27. Holman WL, Kormos RL, Naftel DC, et al: Predictors of death and transplant in patients with a mechanical circu- latory support device: A multi-institutional study. J Heart Lung Transplant 2009;28:44-50.

28. Kirklin JK, Naftel DC, Stevenson LW, et al: INTERMACS Database for durable devices for circulatory support: First annual report. J Heart Lung Transplant 2008;27:10651072.

29. Jahanyar J, Noon GP, Koerner MM, et al: Recurrent device thrombi during mechanical circulatory support with an axial-flow pump is a treatable condition and does not preclude successful long-term support. J Heart Lung Transplant 2007;26:200-203.

30. Takatani S: Progress of rotary blood pumps: Presidential Address, International Society for Rotary Blood Pumps 2006, Leuven, Belgium. Artif Organs 2007;31:329-344.

31. Nishinaka T, Schima H, Roethy $W$, et al: The DuraHeart $V A D$, a magnetically levitated centrifugal pump. The University of Vienna bridge to transplant experience. Circ J 2006;70:1421-1425.

32. Nojiri $C$, Fey $O$, Jaschke $F$, et al: Long-term circulatory support with the DuraHeart mag-lev centrifugal left ventricular assist system for advanced heart failure patients eligible to transplantation: European experiences. J Heart Lung Transplant 2008;27:S245. [Abstract]

33. Tuzun E, Roberts $K$, Cohn WE, et al: In vivo evaluation of the HeartWare centrifugal ventricular assist device. Texas Heart Inst J 2007;34:406-411.

34. Wieselthaler GM, Strueber M, O/Driscoll GA, et al: Experience with the novel HeartWare HVAD with hydromagnetically levitated rotor in a multi-institutional trial. J Heart Lung Transplant 2008;27:S245.[Abstract] 